\title{
Structuring of manual of orientations and technical information for laundries of industries of animal products origin
}

\author{
Vania Eugênia da Silva ${ }^{\mathrm{a}, 1}$, Simone Caldas Tavares Mafra ${ }^{\mathrm{b}}$, Cláudio Lísias Mafra ${ }^{\mathrm{c}}$ e Amaury Paulo de \\ Souza $^{\mathrm{d}}$ \\ a, b, Departament of Home Economics,Federal University of Vicosa, Vicosa, State of Minas Gerais, Brazil \\ ${ }^{c}$ Departament of Biochemistry and Molecular Biology, Federal University of Vicosa, Vicosa, State of Minas \\ Gerais, Brazi \\ ${ }^{d}$ Departament of Engineer Forest, Federal University of Vicosa, Vicosa, State of Minas Gerais, Brazil
}

\begin{abstract}
This study looked for to lift information regarding laundries inserted in industries of animal products origin, to understand the activities developed in the section and to structure a Manual of Planning and Technical Information for laundries to industries of animal products origin, with the intention of subsidizing the planning, structuring and control of this work place, because norms or regulations that supervise don't exist or aid their managers and workers as for the operation of this work place.
\end{abstract}

Keywords: Laundries to industries of animal products origin; Manual; Orientations and technical information.

\section{Introduction}

The search for an environment of work salubrious relationship for several factors, the physical conditions, such as temperature, noise, humidity, brightness and layout of the equipments. The environment should be drifted and structured seeking the best adaptation of the work to the worker. The improvement of the quality of the worker's life contemplates in the services for rendered, but also in the reduction of the indexes of work accidents happened. Besides these, the appearance of occupational diseases is directly linked to the quality of the worker's life, which, brings losses for employee and employer.

The laundry in an industry of animal products origin is configured in this scenery as a support section to the activities developed in the company, tends as main objective the supply of garments properly cleaned and in appropriate amount to the employees' needs that demand them. For the laundry to reach their objectives, it is necessary that the organization and planning of the activities follow a work flowchart to allow good operation.

The work environment in a laundry presents varied risks to their workers, as physical, chemical, biological and ergonomic. According to Silva (2006), the work in laundry is also characterized as monotonous, repetitive and intense, demanding from the workers that these obtain great productivity and a reduced time, under inadequate conditions of work regarding the environment and to the equipments.

In this sense, the elaboration of a manual of information and technical orientations for laundries of industry of animal products origin, it can supply to the professionals of the area and managers of the same ones, offer knowledge and theoretical subsidy aiding in the developed activities, as well as the quality of the services rendered by this section inside of the company, also obtaining improvement of the quality of the employees' life.

In that way, this study aimed at to lift information for, later, to structure a Manual of Orientations and

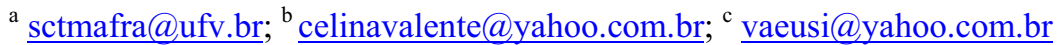


Technical Information for Laundry of industries of animal products origin.

\section{Procedures}

The research was accomplished at two laundries of industries of products of animal origin located in the Area Forest Mineira. The population was formed by workers of the laundry; managers of the laundries; Home Economics, technicians of safety of the work and fiscal of the Service of Federal Inspection (SIF) of the Ministry of the Agriculture, Livestock and Provisioning (MAP) and specialized consultants.

The first phase was characterized by the evaluation of the environment, through measurement of the physical variables.

The noise measurements, illumination and temperature followed Regulatory Roles recommendations (NR's 15 and 17) sockets as reference for the accomplishment of this study. The noise was measured considering the places where there was larger noise propagation, as for instance, close to the centrifuge. The used equipment (digital decibilimeter) it was disposed in the height of the worker's ear. The results were confronted with the established parameters by NR 15 - Activities and Unhealthy Operations.

The limits of tolerance for temperature were also analyzed according to NR 15, with measurement through the meter of Index of Humid Bulb - Thermometer of Globe - IBUTG. It was considered for the calculation of IBUTG an internal environment without solar load.

In the case of the illumination level, the measurements were accomplished with a digital luxímeter, with values taken in the work field where took place the visual task. Already to ventilation and the relative humidity of the air, were measured by an anemometer and a hygrometer, respectively. For ergonomic evaluation, were made direct observations on the routine of the workers' work in order to identify if there were and which the workstations caused them larger discomfort.

The microbiologic analyses were accomplished starting from collected samples of the environment of the laundry in the area of separation of dirty clothes, in the area it cleans and in the area of storage of the processed clothes, and also samples of the dirty uniforms and clean uniforms, ready for distribution, with the purpose of to identify which the possible microorganisms would be present in the environment and the in the uniforms after they be cleaner
For the collection of the samples microbiologic of the clothes the technique of the swab was used, where the microorganisms were collected in the surface of the clothes (it dirties and cleaner) through the $s w a b$ of cotton $0.5 \mathrm{~cm}$ of diameter for $2 \mathrm{~cm}$ of length, being used an applicator of $12 \mathrm{~cm}$ of length. The swabs were sterilized previously by autoclaving to $121^{\circ} \mathrm{C}$ for 15 minutes. After being moistened in saline solution $(0,85 \%)$ the swab forming an angle of $30^{\circ} \mathrm{C}$ with the surface, it was rubbed three times in five areas of $50 \mathrm{~cm}^{2}$ (strips of $5 \times 10 \mathrm{~cm}$ ). Soon after, the $s w a b$ was returned for the tube with the buffered solution $(6 \mathrm{ml})$ sterilized by autoclaving to $121^{\circ} \mathrm{C}$ for 15 minutes. After the immersion, the excess of solution of the $s w a b$ was removed pressing in the walls of the tube.

For the counting of the microorganisms plates were used Petrifilm. That system incorporates an agent soluble like gel, in cold water and tetrazolium indicator to facilitate the visualization and enumeration of colonies (BUGNO, 2005).

The clothes were analyzed in relation to the models and analyses textile of the uniforms of the laundry to identify which the composition of the same ones. Firstly, the touch test was accomplished, that it consists of touching the cloth to identify the texture that characterizes each fiber in a specific way (MARQUES, 2006). Soon after, the test was accomplished of the it burns, that it consists of to burn a piece of the cloth and to identify the fiber, considering the proximity of the fire. In this test, the fabric is put in the fire and, soon afterwards, moved away, observing the characteristic of the ash, odor and coloration of the loosened smoke (CÂNDIDO; VIERA, 2003).

Finally, for the identification of the fibers, the microscopic analysis of the fibers was accomplished used in the making of the workers' of the laundry uniforms.

The third stage consisted of semi-structured interviews with specialized consultants in laundry and Home Economics, to evidence which aspects these professionals consider relevant in the planning and in the structuring of laundries for industry of animal products origin.

\section{Results and Discussion}

\subsection{Risks physical existent in the laundries}

Regarding the risks physical existent, in the laundry "A" it was able to identify: high temperature, 
noise excessive, low relative humidity of the air and ventilation inexistence for the work type.

In the laundry "B" stood out the ventilation deficiency and illumination in all of the workplace, the temperature, noise and humidity if they found in the expected limit for the execution of the activities.

Regarding the ergonomic risks, the two laundries presented similar situations, all the workers executed their activities stand up, with need of inclinations and sprains of the trunk and movements of the arms with great physical effort.

The biological risk found at both laundries were related to a great counting of microorganisms, with prominence for fungi and yeasts and aerobic mesophilic.

\subsection{Analysis of the uniforms of the laundries $A$ and $B$}

The models of the uniforms of the two laundries resembled each other, the differences were in relation to textile composition, being it of the laundry "A" a mixture of fibers that provides larger comfort to the workers. The laundry "B" adopted fabric 100\% synthetic, which can bring discomfort to their users. It is recommended changes in the modelling of the uniform for that it is adapted more to the reality of the task, considering the system man/task, among them: to adopt shirt in mesh that provides larger thermal and lukewarm comfort to the workers; retreat of the collar sport of the shirts, once the same can inconvenience and to disturb in the development of the activities; closing of the front opening of the shirts, with the change of the collar in "V" for a round and solitary collar of the pockets in the pants eliminating possible places of deposition of dirtiness. According to studies found in the literature, the type of more suitable fabric for the production of professional clothes is composed by $67 \%$ cotton and $33 \%$ polyester, once it provides comfort, durability, moderate resilience, good appearance and easy conservation, besides possessing smaller shrink index, when submitted to the successive washes.

\subsection{The workers' of the laundries perception studied in relation to the structuring of the manual}

For the workers interviewees, the structuring of a Manual of Information Technical Orientations for laundries of industries of animal products origin is necessary to aid in the execution of the activities in an appropriate way, guaranteeing sanitary quality starting from the adoption of an efficient processing of the uniforms, improvement of the work routines as well as of the quality of the workers' life, once the environment labor that joins such characteristics will be more adapted for the execution of all of the foreseen activities.

\subsection{Relevant aspects for structuring of a laundry of industry of products of animal origin in the profes- sionals' vision (Home Economics, Manager, Fiscal of the Ministry of the Agriculture, Livestock and Provi- sioning, Technicians of Safety of the Work and Con- sultants of the laundry area)}

For the laundry managers of the studied, they exist environmental factors that they can cause damages to the workers' health in a laundry of industry of animal products origin, highlighting as physical risks the high temperature of the environment, contact with the chemical products, steam of the machines, gas of feeding of the drying ones and transport of wet clothes, ergonomic risks due to the postures assumed by the workers and biological risks by the contact with the unclear uniforms.

For the civil engineer, the laundry is an environment where environmental factors exist (physical, biological and ergonomic) that can cause damages to the workers' health, as the equipments, mainly the centrifuge that it can be open in operation, recommending the choice or equipments adaptation that turn off automatically when open.

For the supervisors of the laundry, the same is an environment where exist environmental factors that they can cause damages to the workers' health, as chemical products, use of the machines, noise, temperature, excessive load and inadequate physical space.

For the district attorney of the Service of Federal Inspection (SIF), of the Ministry of the Agriculture, exist factors that can cause damages to the workers' health, as equipments badly regulated, polluted clothes and still ergonomic factors as the great volume of clothes to be processed in reduced physical spaces and no drifted.

For the consultant, the laundry is an environment that have factors that they can offer risks to the workers' health, as biological, physical and environmental. The biological factors can stand out bacteria, protozoa and helminthes that can cause damages to the workers of the laundry, as for the final product produced by the company, where stands out the environmental risk. As physical factors, the subject of 
high temperatures and humidity that can cause damages to the workers' of this section health highlighted.

For the Technicians in Safety of the Work, the laundry is an environment that have factors that they can cause damages in the health workers', as physical risks, characterized by noise above the acceptable and high temperature; happening of the products of cleaning chemical risks; ergonomic risks, related to the postures adopted by the workers and excess of transported weight; for the same ones, the laundry of an industry of animal products origin doesn't offer biological risks to their workers and the final product.

For the Home Economics, the laundry is also an environment that have factors that they can cause damages in the health workers', for contamination for feces, it urinates, blood or insect. The excessive heat caused by the equipment. Sorts things out incorrect of manipulating the clothes can cause pains and sprains also.

Regarding the that would be necessary for the laundry environment it is considered efficient, for the managers of the companies studied, it was considered, the need of a qualified team with appropriate training, presence of equipments and efficient chemical products, as well as to have the clean uniform in the schedule necessary at shift change, avoiding line and delay of the production.

For the interviewed civil engineer, it is necessary that appropriate ventilation is had and step no slippery. In what says breath to the quality of the rendered service is necessary that it is had qualified professionals to operate efficient equipments.

Still on the theme above, for the supervisors, it is necessary that professionals are had qualified that address the works to be developed at the laundry, taking into account appropriate layout, equipments, specific chemical products for the type of dirtiness of the freezer, certain schedule for delivery of clothes and enough number of workers to the work.

For the district attorney of SIF, it is relevant if to have organization, training, good manipulation practices, technical knowledge, ethics, adaptation of the laundry in all their needs, as size and number of appropriate workers.

For the consultant, to consider an efficient laundry, so much a laundry of products of animal origin, as a hospital laundry, is that these supply uniforms with a low microbiological counting, avoiding a probable worker's contamination that will use this uniform, as well as of the product that will be marketed.

For the Technicians in Safety of the Work, the efficiency in a laundry can be reached is necessary that space appropriate physicist is had, personnel's training with the workers' understanding with relationship norms of safety and layout of the equipments facilitating the work the developed being.

For the Home Economics, the laundry should possess good distribution of the equipment, to avoid the contact of the clothes dirties with the clean, organization, correct use of EPI's and appropriate uniforms, besides the climatization of the environment, to be considered efficient.

Regarding the that is relevant to be observed in the planning of a laundry of industry of animal products origin, for the managers of the companies, it is necessary if it observes the volume of clothes that should be cleaner, the storage capacity, the differentiation among the sections of the laundry, as well as service to the regulatory norms of the Ministry of the Agriculture, that demand that all refrigerating it possesses laundry and Department of Labor, regarding the workers' of the same labor laws.

For the supervisors of the laundries, it is necessary to analyze the regulatory norms of the Ministry of the Agriculture (that demands the presence of the laundry and sanitary barrier), as well as the different wash methods in agreement with the clothes and the dirtiness, always seeking the hygiene of the whole section.

For the district attorney of SIF, it is necessary if to have planning of the flow of the area it cleans and area dirties, use of the chemical products and warranty of good process of cleaner clothes.

For the consultant, it is relevant to observe in the planning of a laundry of industry of animal products origin, it is that guarantees the sanity of the final product, not possessing contamination among area dirties and airs clean and that the equipments if adapt to the reality.

For the Technicians in Safety of the Work, it is relevant to observe in the planning of a laundry, the place where the same will be installed, for not harming the production of the company, as well as the physical structure of the laundry, avoiding crossed contamination and prioritizing physical factors, as ventilation and illumination of the same, it is necessary still to observe the presence of efficient and safe equipment.

For the Home Economics, the physical structure should be observed to guarantee the non contact of the clothes dirties with the clean and the materials of cleaning that will be used.

As for the physical structure, for the administrative managers, this should possess mainly differentiation 
among area cleans and area dirties and still a planning the long term, so that it holds the growth of the company, and the demand for the services rendered by the laundry.

According to the interviewed civil engineer, the physical structure of the laundry it should possess separation among area dirties and area cleans. The flow of the clothes should follow the itinerary done by the workers from entrance in the company until the change of clothes for the uniform already cleaner, avoiding dirtiness come back of the street as soon as contaminates the dependences of the company.

For the supervisors of the laundry, the physical structure of the laundry should plead physical and airy space, drifted in agreement with the flowchart of developed work so that it avoids the excessive transport of weight, avoiding to waste of time during the process and so that it can't control the crossed contamination.

For the district attorney of SIF, it is necessary if to have appropriate to the demand, easy access to the workers and delivery of the clothes, presence of clean area and area dirties and planning so that the obstacle flow is avoided.

For the consultant, the physical structure it should prioritize the contact inexistence among area cleans and area dirties, avoiding against-flow.

For the Technicians in Safety of the Work, the physical structure of the laundry should possess division in dirty areas and cleans area avoiding the contact of the unclean clothes no with the remaining of the laundry, should also seek to reach situation of comfortable work with relationship physical variable providing ventilation and illumination adapted, creating an ergonomically environment appropriate to the development of the activities.

For the Home Economics, the structure should be divided in area dirties and clean, without there is contact of the same ones, besides bathroom for area dirties and stock room and dilution of products.

In relationship the work flow in the laundry, the managers evidenced a progressive flow of the activities to the be developed, with presence of dirty area and area cleans delimiting the contact of unclean clothes with clean clothes.

For the managers of the laundry, the work flow should be lineal, avoiding the workers' excessive wear and tear and people's of the area contact cleans with the area dirties.

For the district attorney of SIF, the flow should assure that there is not the people's of the area access dirties for the area cleans, reducing the against-flow risk.

For the consultant, the work flow in a laundry of industry of animal products origin, should assist the demand of the industry of shifts differentiated of workers, avoiding the delay of delivery of clean clothes and possible delay of the operation of the section of production of the company.

For the Technicians in Safety of the Work, the work flow should be to avoid the area crossing dirties with area cleans, following the operational procedure for cleaner clothes.

For the Home Economics, the flow should be made as of a hospital laundry, following their procedures and sanitary hygienic control.

When investigated on the importance of the laundry for the institution, the administrative managers of the companies told, that the laundry is of fundamentally an importance for the good operation of the company, because it guarantees the final quality of the product, with the workers' safety always use cleaner uniforms and guaranteeing the change of uniforms when necessary.

According to the interviewed civil engineer, the laundry has the importance of avoiding contamination, for having the warranty correctly of being offering to the cleaner uniform worker, assisting to acceptable limits of contamination. Another importance of the laundry is the organization checked to the company, once all the workers are uniformed.

For the supervisors of the laundries, the laundry has the importance of contributing for the improvement of the final quality of the product marketed by the company, impeding that don't happen risks of contamination of the manipulators' garments.

For the district attorney of SIF, the laundry assumes great importance in relation to the company, once it avoids that the workers wash their uniforms home, possessing larger control of whole the wash process avoiding like this the risk of contamination.

For the consultant, the laundry is fundamental for the company, once starting from this section the sanity of the product can be guaranteed.

For the Technicians in Safety of the Work, the laundry possesses great importance for the company, to maintain the uniform in an acceptable pattern for the production, considering safety's norms and of cleaning in general.

According to the Home Economics, the laundry assumes the importance of guaranteeing clean clothes, free from infection and for a smaller cost, 
contributing to the good operation of the whole business structure.

For the managers of the studied companies, that is waited of the laundry is that washes and cleaner the uniforms in a correct way, maintaining a visual microbiological pattern of quality of the processed uniforms.

For the supervisors of the laundries, what waits of a laundry, it is the efficient operation that assists the workers of the production with clean clothes the on time certain for the production so that it minimizes the excessive wear and tear of the workers, of the products and uniforms.

The district attorney of SIF hope a laundry has efficiency to assist the demand of the company appropriately regarding the volume and the capacity of cleaner of the clothes, prioritizing the quality of this process.

The consultant interviewee wait that the laundry given the processed uniforms in appropriate way of the sanitary point of view can be hospital, industrial or maidservant, always prioritizing the cleaner of the same.

For the Technicians in Safety of the Work, which one should wait of a laundry is that the uniforms are in appropriate conditions of use in the aspect of cleaning and provide physical and psychological condition of work to the worker.

The Home Economics hope a laundry supplies clothes in amount and appropriate hygienic quality, assisting the needs disputed by the company and get a control and reduction of the costs of the same.

Regarding the aspects that it considers relevant in the planning and laundry structuring for industries of animal products origin, for the managers, a long term planning should be had holding the possible growth of the whole business structure, including in this place planning, of the space, destination and quality of the water used at the laundry, care with the chemical products and looking for support technician and theoretical structure of the activities to the be developed.

In what refers to the planning and structuring of laundries for industries of animal products origin, the civil engineer gives importance to the cleaner process in order to if avoids contamination, as well as to the controlled flow and addressed of workers' entrance in the company directly to the dressing room to change the uniform and take a shower.

For the supervisors of the laundries, the relevant aspects in the planning and structuring of a laundry, it is the organization of whole the space, from physi- cal space, equipments and work routines, prioritizing means of avoiding the against-flow of the activities developed in this adapts.

The district attorney of SIF considered relevant in the planning and structuring of laundries in industries of animal products origin, the workers' training, good operational organization, facilities and appropriate equipments to the demand.

For the consultant, it is relevant to observe in the planning and structuring of a laundry of industries of products of animal origin, that the same doesn't allow their workers' contamination and of the processed the uniform and, still, that she guarantees an environmental safety, not contaminating with factors probable chemical and biological courses of rivers and close streams.

Regarding the planning and structuring of laundries, the Technicians in Safety of the Work suggest that it owes if it takes in consideration, the functionality, facilitating the process of work, layout of the equipments, physical space to assist the current and future demands of the company.

For the Home Economics, the planning of a laundry should consider a good distribution of the equipment, to avoid the contact of the clothes dirties with the clean, organization, correct use of EPI's and appropriate uniforms, besides the climatization of the environment.

As for the aspects that should be present as recommendations or as demands in the manual of information and technical orientations for laundry of discount industry and processing of animal products origin, the managers emphasized the indication of the main equipments with the loads, work flow, use of the chemical products and their destinies for each type of dirtiness, information on woven and usability and physical space.

For the civil engineer the aspects that should be present as recommendations or demands in a manual of information and technical orientations for laundry of discount industry and meat processing, the interviewee considers that it should contemplate means of avoiding the crossed contamination, motivating lineal activities avoiding against flow.

Regarding the structuring of a manual of informations and orientations, the supervisors of the studied laundries emphasize the need of information to respect developed operational processes, use and indication of the chemical products, physical space and dimension in agreement with the volume of processed clothes and training of personal. 
For the district attorney of SIF, it should be present as recommendations or demands in a manual of information and technical orientations, the themes the regarding the use of addictive and approved detergents in agreement with the rules of Ministry of Health and Ministry of the Agriculture. To contain still information regarding the dynamics of operation of the same, avoiding against flow and possible contamination of this adapts.

For the consultant interviewee, it is necessary to be present in the Manual of Technical Information for laundries of industries of animal products origin, that the laundry is faced by the company as a strategic and primordial section, where can will guarantee the safety and sanity, so much of the worker that works in the same and in the section of production of the company, as of the product that will be marketed. Another important point to be related is the environmental subject, regarding the infrastructure that permeates the electric power consumption, consumption of water and necessary equipments for operation. As well as it is necessary that in this section has workers to develop the activities foreseen for the same, and that it doesn't possess workers that were not fit in other sections of the company and they were transferred to the laundry. It is necessary still to contemplate aspects related to the equipment, regarding the capacity and way of operation of the same.

For the Technicians in Safety of the Work, the Manual should contemplate information regarding operational procedures of each activity, controls of the developed activities, arrange appropriate physicist with division of areas, equipment that assists the productivity and safety, chemical products of quality and necessary equipments of individual protection to the execution of the activities.

According to the Domestics Economists, the Manual should possess as content norms and conducts of the section, eminent biological risk to the developed activities. Correct dosage of products, correct use of EPI's, annotates technique of the equipments and products.

\section{Conclusions}

The structuring of a Manual of Information and Technical Orientations for laundries of industries of animal products origin important front is shown to the reality of the section. Therefore, for this structuring it was necessary to look for information regarding the subject, that it guided the execution of the study, it was not found specific literature on the subject, being necessary the search for sources that approached laundries where their activities resemble each other to developed them in a laundry of industry of animal products origin, besides being necessary to know the work routines developed, to understand how the organization of the section is.

The studied laundries possessed great differentiations in their physical structures and work routines.

Regarding the present risks in the studied laundries, it was possible to identify several risks in both realities, as physical risks was high temperature, noise excessive, low humidity and ventilation inexistence, deficiency illumination. Regarding the ergonomic risks, the two laundries presented the same conditions, all the workers executed their activities stand up, removing and putting clothes in the machines that it demanded inclinations and sprains of the trunk and movements of the arms with great physical effort. The biological risk was found at both laundries great counting of microorganisms, detaching fungi and yeasts and aerobic mesophyll. In spite of finding the presence of microorganisms it can be considered the processes of cleaner efficient, because they didn't cross the limit of UFC used as reference in the research. Important to emphasize the inexistence and it doesn't complete separation of the dirty and clean areas found at the laundries, what can contribute so that the contamination microbiological is significant and harmful to the workers and the final product by the companies.

All the workers' of the companies laundries cleaner your uniform. In both realities differentiations existed regarding the colors, what facilitated in the separation for dirtiness.

The models of the uniforms of the two companies resemble each other, possessing details as pockets and lapels that hinder in the elimination of dirtiness, they possess differentiation regarding textile composition.

It is necessary that feels larger attention and importance to the laundry inserted in an industry of animal products origin, once their activities are unhealthy to their workers, what can modify the life quality. It is necessary that manager has theoretical and practical knowledge about the routines, particularities and objectives, improving like this the whole section.

For the workers and professionals interviewees, the structuring of a Manual of Information Technical Orientations for laundries of industries of animal products origin is necessary to aid in the execution of 
the activities in an appropriate way, guaranteeing sanitary quality starting from the adoption of an efficient processing of the uniforms, improvement of the work routines as well as of the quality of the workers' life, once the environment labor that joins such characteristics will be more adapted for the execution of all of the foreseen activities. Like this, the development of this study was made relevant, because the final product, which is, the Manual of Orientations, is put as a contribution for the section studied in what refers to planning, structuring, implantation and development, in efficient and effective way of the developed work and guaranteeing larger satisfaction and comfort to the worker.

\section{Acknowledgement}

The research receives financial support of National Council of Advice of Research and Technological Development (CNPq) and Foundation of Help to the Research in the State of Minas Gerais (FAPEMIG).

\section{References}

[1] Brasil, Ministério do Trabalho e Emprego. NR-15, Atividades e Operações Insalubres. Available on <http://www.mte.gov.br/Empregador/segegsau/Co missoes-

Tri/ctpp/oquee/conteudo/nr15/default.asp $>$. Access on: May 2009

[2] Brasil, Ministério do Trabalho e Emprego. NR-17, Ergonomia. Available <http://www.mte.gov.br/Empregador/segegsau/C omissoes-

Tri/ctpp/oquee/conteudo/nr17/default.asp $>$. Access on: May 2009.

[3] Bugno, Adriana, Almodovar, Adriana A. B.; Pereira, Tatiana C.; Pinto, Terezinha de Jesus. Aplicabilidade de Petrifilm na enumeração de bactérias e fungos em drogas vegetais. Rev. Inst. Adolfo Lutz. p.20-24,2005.

[4] Cândido, Índio; Vieira, Elenara Vieira de. Lavanderia hoteleira: técnicas e operações. Caxias do Sul: Educs. $2003.181 \mathrm{p}$.

[5] Marques, M.A.R. Análise e percepção do processo de higienização de roupas profissionais em indústrias alimentícias. Viçosa: UFV, 2006. Dissertação (Mestrado em Economia Doméstica). Universidade Federal de Viçosa, 2006.

[5] Silva, V.E. Qualidade de Vida em uma lavanderia de indústria de abate e processamento de carne, pela avaliação das condições dos riscos ambientais e sócio-culturais. Viçosa: UFV, 2006. 159f. Dissertação (Mestrado em Economia Doméstica). Universidade Federal de Viçosa, 2006. 